\title{
BMJ Open The Australian primary healthcare experiment: a national survey of Medicare Locals
}

\author{
Suzanne Robinson, ${ }^{1}$ Richard Varhol, ${ }^{1}$ Vijaya Ramamurthy, ${ }^{1,2}$ Melissa Denehy, ${ }^{1}$ \\ Delia Hendrie, ${ }^{1}$ Peter O'Leary, ${ }^{1}$ Linda Selvey ${ }^{1}$
}

To cite: Robinson S, Varhol R, Ramamurthy V, et al. The Australian primary healthcare experiment: a national survey of Medicare Locals. BMJ Open 2015;5: e007191. doi:10.1136/ bmjopen-2014-007191

- Prepublication history for this paper is available online. To view these files please visit the journal online (http://dx.doi.org/10.1136/ bmjopen-2014-007191).

Received 13 November 2014 Revised 2 March 2015 Accepted 3 March 2015

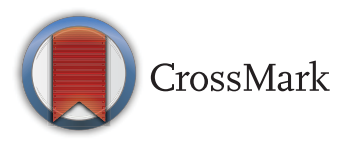

${ }^{1}$ Faculty of Health Sciences, Curtin University, Perth, Western Australia, Australia ${ }^{2}$ Western Australia Department of Health, Perth, Western Australia, Australia

Correspondence to Dr Suzanne Robinson; Suzanne.robinson@curtin. edu.au

\section{ABSTRACT}

Objective: The objectives of this study are to evaluate the development and implementation of Medicare Locals as new primary care organisations and consider the implications of these findings for the wider challenge of strengthening primary healthcare in Australia and internationally.

Design: National survey of Medicare Locals which involved the use of content analysis and a descriptive survey tool.

Setting: 61 Medicare Locals in Australia.

Participants: The survey was distributed electronically to all 61 Medicare Local Chief Executive Officers (CEOs) between October and December 2013.

Main outcome measures: The research was interested in exploring the following areas; the form and function of Medicare Locals; the confidence and capacity of Medicare Locals to perform against their objectives around population planning and system integration; their ability to engage relevant stakeholder groups; and the barriers and facilitators to reform.

Results: A total of $43(70 \%)$ of Medicare Locals completed the survey with representation from six of the eight Australian states and Territories. Results suggest differences in the form and function of the Medicare Local organisations and considerable diversity in the implementation of Medicare Local organisations across Australia. This diversity and lack of guidance from government impacted on the overall success of the reform. Other barriers to reform included difficulties in stakeholder relationships and limited incentives (financial and other) to drive and influence change. Conclusions: Findings from this study produce important insights for primary care reform in Australia; and internationally it adds to the growing body of knowledge around primary care reform.

\section{INTRODUCTION}

The Australian health system performs well compared to other countries in the Organisation for Economic Cooperation and Development (OECD). ${ }^{1}$ However, it does face a number of major challenges relating to equity, including issues around access and

\section{Strengths and limitations of this study}

This is one of the first independent published studies that provides evidence on the development and implementation of the Medicare Local (ML) reform in primary care in Australia MLs.

- It provides information that will be of importance to the development of Primary Health Networks in Australia and for development of primary healthcare internationally.

- The response rate is high for a study of this kind.

- It explores the views of ML executives, with no intent to survey wider stakeholder groups.

- It was undertaken at an early stage in the implementation of MLs, and during a period of uncertainty as to Government's continued policy commitment to these meso-organisations.

- No follow-up investigation was undertaken.

lower life expectancy; variations in clinical practices; and quality of care between regions. Populations living in rural and remote areas have less access to healthcare resulting in poorer health outcomes than the national average, with even greater discrepancies evident for indigenous populations. ${ }^{2}$ Australia, like other nations, faces the pressures of an aging population; rise in chronic illness; and limited resources. While the system delivers a high level of patient care at this time, its future sustainability and performance has been questioned, with the system regularly criticised for its fragmentation and lack of integration between sectors and a provider rather than patient centric focus. ${ }^{3}$

Evidence suggests that an integrated system of healthcare with a strong primary healthcare focus, leads to a more efficient and effective healthcare system with improved patient outcomes and experiences. ${ }^{4-6}$ Over recent years, a number of countries have attempted to establish structures and organisations that support the primary healthcare sector. The introduction and devolution of power to regional meso-level primary healthcare 
organisations in countries such as UK, Canada and New Zealand, has brought demonstrated improvements in efficiency and quality of care, and helped to facilitate better health system integration. ${ }^{7}{ }^{8}$ Meso-level organisations are positioned between government (macro-level) and micro-level groups and individuals who deliver services. ${ }^{9}$ The structure and governance arrangements of meso-level organisations varies across jurisdictions, as do their purchasing and procurement responsibilities.

One of the major challenges in undertaking primary healthcare reform is shifting the balance of power and resources from secondary to primary healthcare. Primary healthcare is typically the poor relation in comparison to hospitals that attract greater political, resourcing and public support. ${ }^{10}$ There are a number of reasons for this disparity, including the fragmented nature of primary healthcare providers and their lack of a collective authority and position as policy and resource stakeholders. ${ }^{11}$ Traditionally general practitioners, operating as small and independent business operations, have less tendency or capacity to mobilise their collective powers, compared to hospital specialists for whom this comes more naturally, operating in larger clinical teams with more significant resources. ${ }^{11}$ These modes of operation are likely to have arisen from traditional delineations in the level of care, with general practitioners seen as providers of less complex healthcare than their hospital-based colleagues. In recent decades, however, the rising incidence of chronic disease and a number of patients with comorbidities, and enhancements in medical technology has dramatically changed the primary healthcare landscape. There is now a both a pressing need and opportunity to meet and manage healthcare needs earlier, and with greater effectiveness, in primary healthcare settings.

Studies have demonstrated that for primary healthcare reform to be effective, it needs to be undertaken as part of a whole system change, involving support and leadership from clinicians, especially general practitioners. ${ }^{12} 13$ For example, a study by McCafferty et $a l^{14}$ which evaluated primary healthcare reform in the UK noted some important aspects for success including the need for a system-wide approach with appropriately aligned objectives and incentives, along with the coordinated support and management of organisational cultures. Evidence also suggests that such system level reform requires strong and effective leadership to navigate the often difficult political terrain, as well as strong skills in relationship management and coalition building. ${ }^{12}$

In Australia, the Commonwealth government embarked on primary healthcare reform in $2010^{3}$ through the establishment of Medicare Locals (MLs), meso-level organisations whose role was to build on the work of existing Divisions of General Practice and further strengthen primary healthcare. ${ }^{15} 16$ The 61 MLs established sought to have a stronger focus on population health and planning than their predecessors, with an overarching aim to enable "better access to services, improved local accountability and transparency, greater responsiveness to local communities and provide a stronger financial basis for our health system into the future." ${ }^{17}$ However, a change of government in 2013 has seen the disbanding of MLs. The Government's decision to disband MLs followed the recommendations of the Horvarth review ${ }^{18}$ which suggested that in their current form MLs were not appropriate or effective to successfully achieve their strategic aims (p.9). The Government has since introduced a new form of meso organisation, Primary Health Networks (PHNs) which are fewer in numbers (moving from 61 MLs to 30 PHNs). ${ }^{19}$

Prior to being disbanded, MLs were relatively new organisations, some had only just reached their first anniversary when the disbandment was announced in $2014 .^{20}$ As such there has been little opportunity to evaluate and gather information and intelligence on their development and implementation experiences, and there is little by the way of independent evidence to help inform current and future primary health care policy development in Australia.

This paper reports findings from a study of MLs across Australia, aimed at providing a national picture of ML models and practices. The paper also considers the implications of these findings for the wider challenge of strengthening primary healthcare in Australia and internationally.

The research was designed to explore the following:

- The form and function of MLs;

- The confidence and capacity of MLs to perform against their objectives around population planning and system integration;

- Their ability to engage relevant stakeholder groups; and

- The barriers and facilitators to reform.

\section{METHODS}

Our national study established a detailed mapping of all 61 MLs using content analysis and a descriptive survey tool that was distributed to all MLs. Descriptive surveys offer a method of data collection that is designed to measure attitudes, knowledge and behaviour in samples of the population of interest. ${ }^{21} \mathrm{~A}$ desktop review of webbased material and other published documentation was also conducted to map the formation, structure and activity of each ML with a specific focus on the following details of ML operation:

- Board formation (number and types of professionsincluding the number of General Practitioners (GPs));

- Formation and structure of core ML staff;

- Stakeholder engagement, population planning and needs assessment; and

- Priority areas for development.

The descriptive survey was designed and developed in consultation with ML representatives to ensure it was relevant and clear in terms of the questions being posed. The survey posed a series of questions relating to the different aspects of MLs including the role and remit of MLs; capacity and confidence in population health planning; 
ability to engage with stakeholder groups; and facilitators and barriers to success. The survey tool encompassed a range of tick boxes, likert-scaled responses and also included space for free text responses that aimed to capture additional information and issues that were not covered by the quantitative questions.

A copy of the survey tool can be obtained from the authors on request. The survey was distributed electronically to all 61 ML Chief Executive Officers (CEOs) between October and December 2013 with the aim of exploring the views of this specific stakeholder group. Returned data were transferred into Excel for ease of analysis. Descriptive statistics are used in this paper to report quantitative responses. Free text responses were categorised into emergent themes and is reported alongside the quantitative data, to assist in terms of the explanatory power of these findings.

\section{RESULTS}

A total of 43 (70\%) MLs CEOs completed the survey, with representation from six of the eight Australian States and Territories (the Australian Capital Territory and Northern Territory are not represented in the data). With the exception of Queensland, which had $55 \%$ of MLs represented, the remaining five respondent states had between $64 \%$ (Victoria) and $100 \%$ (Western Australia) participation in the survey (table 1). By way of regional representation there was representation from across all of the Commonwealth government peer groupings, which are based on remoteness and socioeconomic status of populations. These groupings

\begin{tabular}{lc} 
Table 1 & Participation by state and peer groupings \\
\hline & Per cent \\
\hline State & 0 \\
Australian Capital Territory & 71 \\
New South Wales & 0 \\
Northern Territory & 55 \\
Queensland & 80 \\
South Australia & 100 \\
Tasmania & 71 \\
Victoria & 100 \\
Western Australia & \\
Peer groupings* & 63 \\
Metro 1 & 92 \\
Metro 2 & 43 \\
Metro 3 & 88 \\
Regional 1 & 69 \\
Regional 2 & 80 \\
Rural 1 & 50 \\
Rural 2 & \\
\hline *Metro 1: high urban density, higher socioeconomic status; Metro \\
2: medium urban density, medium socioeconomic status; Metro 3: \\
low urban density, lower socioeconomic status; Regional 1: outer \\
urban areas, middle socioeconomic status; Regional 2: mostly \\
non-metro urban and regional areas, middle socioeconomic \\
status; Rural 1: distant from metro cities, with diverse \\
socioeconomic status; Rural 2: mostly large remote areas, middle \\
or lower socioeconomic status. \\
\end{tabular}

include Metropolitan 1-3; Regional 1-2 and Rural $1-2 .{ }^{22}$ As table 1 demonstrates all but two peer groupings (Metro 3, 43\%; Rural 2, 50\%) had over 60\% representation. While the results outlined in table 1 demonstrate fairly good coverage across ML and the different peer groupings, the lower response rates tended to be in areas across Metro (Metro 3) and Rural (Rural 2), areas that have populations of lower socioeconomic status and density.

\section{Form and function of MLs}

There was a disparity in the composition and functionality of ML organisations, with board structures and activity of MLs being two notable differences. In terms of board membership, the Commonwealth government requirement included: a skills based rather than a representative Board; no one profession should dominate; and a minimum of seven members. Our desk top review which included information for all 61 MLs identified that $45 \%$ of MLs had 9 members, $28 \%$ had 7 and $27 \%$ had 8 members. The number of general practitioners serving on ML boards ranged from one to five, with an average of three. The number of GP board members tended to increase with urbanisation. The number of executive staff working within MLs ranged from 1 to 14 , with an average of five executive staff per ML. Forty-one per cent of MLs had at least one employee categorised in a needs assessment or planning role. Those MLs with no needs assessment and planning staff tended to be more prevalent in remote areas (the percentage of MLs that reported they had no needs assessment staff ranged between $21.1 \%$ in Metro areas to $30.8 \%$ in rural and $42.9 \%$ in remote areas). For a number of MLs who reported having no needs assessment staff, some or all of their needs assessment work was commissioned to external organisations-including universities and national and international consultancy organisations.

The number of staff employed in MLs ranged from 7 to 129 (information relating to total staff employment was only available for $50 \%$ of MLs). The majority of MLs had commissioning and provider functions although the level of provider functions and activity varied between organisations. The types of roles undertaken by MLs varied, however, all MLs participating in the survey mentioned their focus around population health planning and coordination; with the majority of respondents referring to their activity in developing after-hours services; mental health provision, immunisation and promotion of eHealth records. Some MLs noted the continuation of work conducted by their predecessors (DGP), which often included the expansion of chronic disease management.

\section{Confidence in planning and coordination functions}

The survey also sought to explore the capacity and confidence of MLs staff in undertaking the planning and coordination role. 
When asked about their confidence to undertake planning activity, $62 \%$ of respondents suggested that they felt their ML was highly confident in undertaking planning activity, while all the remaining respondents suggested they felt moderately confident with their ML's ability to undertake this role. Additionally, 37\% of respondents suggested they felt highly confident in the ability of the ML to undertake a coordination role, $45 \%$ suggested they were moderately confident, while $18 \%$ suggested they had low confidence in the ML's ability to undertake the role. Respondents tended to have less confidence in their ML's ability to respond to emerging primary healthcare priorities with $36 \%$ of respondents feeling highly confident; $46 \%$ moderately confident and $18 \%$ expressing low confidence.

\section{Needs assessment}

One of the strategic objectives of MLs was to identify the health needs of local areas and develop locally focused and responsive services. It was mandatory for all MLs to undertake a needs assessment exercise. Our survey explored various aspects relating to the undertaking of the needs assessment including: the types of data sources used to inform ML needs assessment; resources and support utilised; use of techniques such as spatial planning; and how information generated from need assessment exercises was used.

The types of information used by MLs to inform needs assessment processes are outlined below at table 2, with the main source of information being data from the Australian Bureau of Statistics.

Respondents were asked to rate their views on the usefulness of the different data sources in helping them formulate their needs assessment, with 0 being 'not at all useful' through to 10 which represented 'very useful' (figure 1). All data sources scored above 5, with the most useful sources being the Australian Bureau of Statistics, survey data obtained by the MLs themselves, and the Public Health Information Development Unit PHIDU). Eighty per cent of respondents believe that there is a need to improve access to relevant data sources, especially in relation to primary care data from general practice and allied health, with a number of participants outlining the need for more high quality primary care data.

Table 2 Data sources used to inform needs assessment

\begin{tabular}{ll}
\hline Data sources & Per cent \\
\hline Australian Bureau of Statistics & 95 \\
Other state health data & 86 \\
Existing data from local organisations & 84 \\
Public Health Information Development Unit & 83 \\
Australian Institute of Health and Welfare & 81 \\
Own survey data & 79 \\
Local GP data & 60 \\
National Health Performance Authority & 51 \\
Australian National Preventative Health Agency & 37 \\
\hline
\end{tabular}

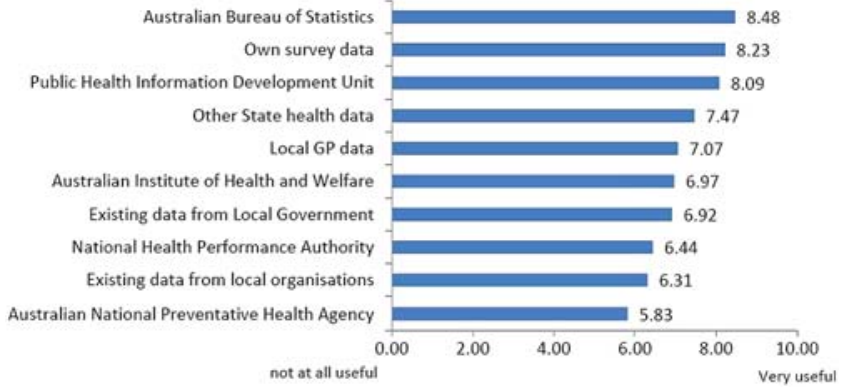

Figure 1 Average respondent rating of the usefulness of different data sources in helping them formulate their needs assessment.

All respondents suggested that they would be using the information generated by their needs assessment to inform population-based planning and priority setting, and facilitate primary healthcare planning discussions with wider stakeholder groups. In addition, other activities being informed by the needs assessment included: pre-evaluation and post-evaluation of service provision; development of specific health plans; benchmarking and comparisons of year on year activity; resource allocation decisions and the formulation of shared health and well-being plans.

Seventy per cent of participants suggested they had used spatial information to inform their population health planning. This tended to be used to map services against need and highlight any gaps in service provision. When asked about barriers faced by MLs when conducting needs assessment, 49\% highlighted issues around resources and data (with particular reference to the provision of more granular (Statistical Local Area (SLA)) level data), $38 \%$ mentioned issues around time constraints and reporting timelines, while $18 \%$ referred to the lack of qualified staff to conduct the analysis.

The study also examined the priority areas identified by MLs in their needs assessment. The major priority areas tended to be closely aligned with Commonwealth programme priorities including: indigenous health; mental health; aged care; chronic disease; eHealth; and after hours services. Figure 2 shows the breakdown of the main priorities identified across the ML peer

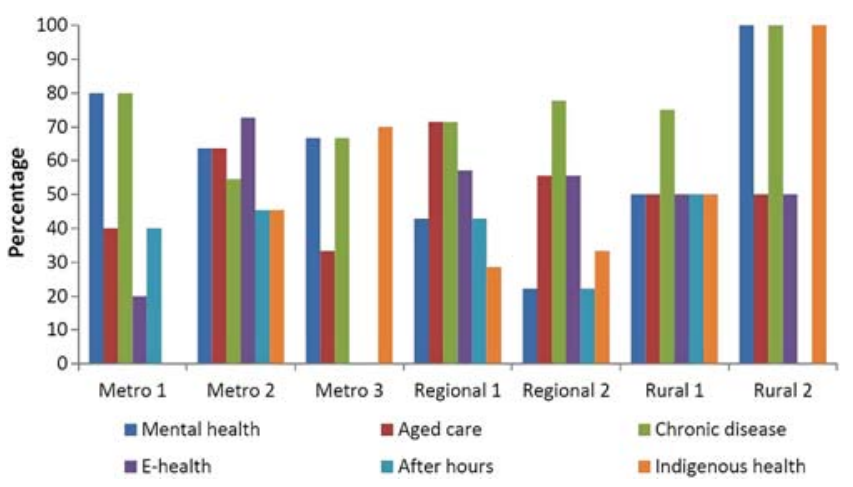

Figure 2 The main priority areas identified by Medicare Local peer groupings. 
groupings. Over $50 \%$ of respondents in each peer group identified chronic illness as a major priority area. With the exception of MLs in Regional 1 and 2 areas, mental health was highlighted as a major priority area, with over $50 \%$ of MLs for each grouping highlighting as a priority. Around $30 \%$ of MLs stated that their priority areas were focused on addressing equity and access issues, and on service mapping and service gap analysis; especially in relation to allied health and community support services. Over $20 \%$ of respondents suggested that one of their priorities was to provide services if there was a need or gap in current provision.

\section{Stakeholder engagement}

To achieve their strategic objectives, MLs were required to engage with a number of stakeholder groups from across the health system. Respondents identified a variety of different stakeholder groups whom they had some level of formal involvement with. At the time of the survey, respondents suggested that they tended to have strongest engagement with the department of health and ageing and less engagement with consumers (figure 3). Going forward, MLs indicated that their stakeholder engagement activity would become more focused on general practitioners (73\%); local hospital networks (60\%); allied health professionals (43\%); and consumers $(30 \%)$. The main barriers to collaboration highlighted by respondents included: competing stakeholder interests (70\%); perceived lack of clarity about the role of MLs (on the part of the external organisation) $(65 \%)$; unrealistic timeframes for delivering objectives $(60 \%)$; conflicting expectations among stakeholders (47\%); and unrealistic expectations among stakeholders $(40 \%)$.

The survey asked respondents about their views on what needed to occur to improve collaboration across the local health system. The main themes generated included:

- Structural and policy change;

- More policy direction from the Commonwealth government;

- More connectivity between MLs;

- Relationship building with stakeholders-(trust); and

- Increased levers and incentives.

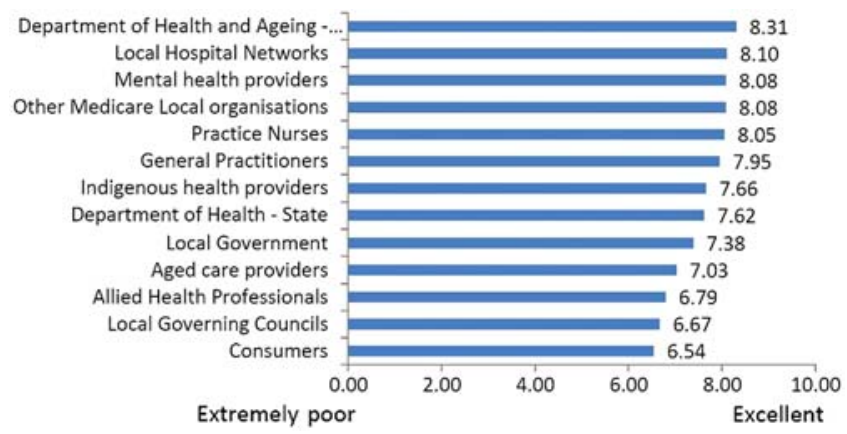

Figure 3 Diversity and amount of stakeholder engagement.
The structural and policy change elements tended to relate to the structural barriers around funding and government structures, as one respondent noted:

Greater planning across the levels of the health system is needed. An effective health system does not work in silos and at present this is happening as a result of funding and state and federal objectives and deliverables. All levels of the health system need to work together to ensure there is a focused response to health across the continuum.

The perceived lack of policy direction from the Commonwealth government and the lack of connectivity between MLs, were also identified as barriers to collaboration. This view was held by a number of respondents who suggested that a lack of national direction had led to considerable variation in the form and function of MLs (ie, the way ML policy was being interpreted and implemented at local level), this being further exacerbated by a lack of connectivity and competition between MLs. Some of the key comments made included:

The lack of national policy or plan leads to 61 variations with little demonstrative health outcomes for consumers.

Stronger Commonwealth leadership and direction on the interface between primary and acute care.

More collaboration and co-ordination across MLs would reduce duplicate effort and stop confusion of external stakeholders

Respondents also referred to the need to build relationships and alliances with key stakeholder groups. The word 'trust' was used by a number of respondents, which generally referred to the lack of trust and a scepticism about the role of MLs in some stakeholder groups.

There is a lack of trust in relation to our role and our intentions, this often links to the lack of understanding about our role and we need to work with stakeholders to improve this.

It is early days but we need time to strengthen relationships-after all, that is what it's all about.

Another limitation to collaboration and integration identified by respondents related to a perceived lack of levers and incentives to change and shape behaviour. This brings to light the difficulties faced by MLs in leading and coordinating activities with a view to achieving their strategic objectives, in the absence of any real authority or power. As one respondent noted:

MLs are small players in the primary healthcare arena and have little in the way of reform levers (eg, financing, standards of practice etc).

To evaluate what other implementation challenges MLs encountered, the study asked respondents to 
identify what other key barriers MLs faced in regard to achieving their strategic objectives. Figure 4 outlines some of the main barriers, with lengthy reporting requirements identified as the main barrier to achieving objectives, followed by insufficient funding.

While respondents identified a number of barriers around the realisation of strategic objectives-when asked if their activities had led to improved service coordination and greater responsiveness to the needs of the local community, $92 \%$ of respondents thought they had. Respondents provided a range of examples pertaining to the MLs role in coordination and integration of services. A number of respondents mentioned work related to developing clinical pathways, which involved linkages between acute and primary care. Some respondents mentioned their role as being 'part of the wider social determinates of health', and involved working with local government agencies assisting with housing and educational needs. In relation to their role, respondents used phrases such as: undertaking shared planning; raising awareness; identifying different ways of working; improving access to services especially for disadvantaged groups; and improved service planning and coordination. However, while respondents noted positive advancements around their strategic objectives, it was noted it was relatively early days in terms of provision of evidence around success.

\section{DISCUSSION}

As the new PHNs are being established, there is little evidence about the success or otherwise of their predecessor organisations to guide policy formulators and implementers going forward. This paper seeks to address this gap, reporting evidence from one of the first national studies on the implementation experiences of MLs across Australia. It is clear that as with all new organisations, much of the early work and energy around MLs was dedicated to the development of organisational and governance structures. Evidence suggests that it can take around 18 months for major organisational restructures to have meaningful impacts. ${ }^{23}$ With many of the organisations surveyed only being in their first year of establishment, the snapshot captured through our study is of meso-level organisations at the infancy stage of their evolution. Furthermore, the survey

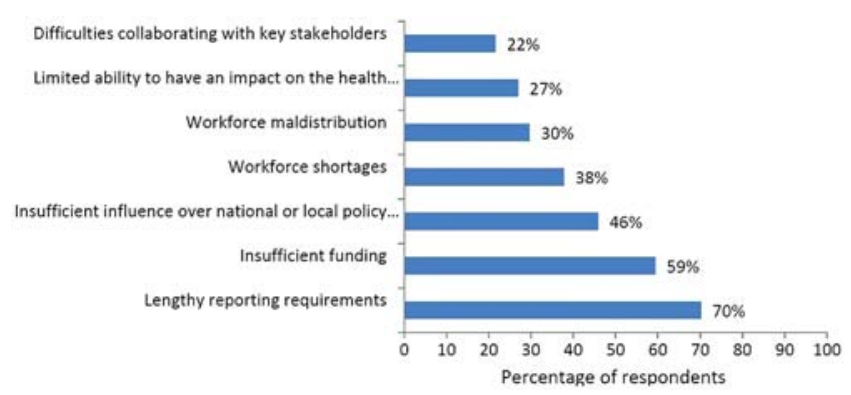

Figure 4 Barriers to achieving strategic objectives. occurred during a period of policy uncertainty, with the incoming Government's continued policy commitment to these meso-organisations being unclear at the time. Notwithstanding these limitations, the survey results do identify some important implementation issues that have very relevant implications for the new Australian PHNs who are tasked with further driving the primary healthcare reform agenda, and for meso-level organisations in other primary care systems.

The survey evidence clearly shows a diversity and difference in the form and function of MLs. The key policy issue is that in establishing future meso-level organisations, more thought needs to be given to striking a better balance between being local and responding to local needs, and retaining some consistency between organisations. On the latter, the lack of a more coordinated and integrated approach was likely to have been at least partly due to insufficient national policy direction, which was noted as being problematic by some ML respondents. Our study also identified the competitive nature of MLs with differences in the make-up of boards and in their role, with some undertaking a predominantly commissioning role while others undertaking a mix of commissioning and provider roles. While competition and variation is not necessarily a bad thing, particularly where variation is an intentional part of design to enable better meeting of local needs, in the case of MLs, the variation was not adequately accompanied by an integration of reform effort at a national level.

At this infancy stage of reform, when providers and consumers were still learning about the role of MLs, the excessive disparity in implementation served to confuse stakeholder understanding around the role of MLs and thus became a barriers to the overall success of the reform. These findings suggest that the Commonwealth government needs to be more involved, particularly during the establishment stage of primary healthcare reform, to monitor implementation divergences and facilitate system-wide harmonisation where appropriate, in cooperation with meso-level organisations and key policy stakeholders. In undertaking primary healthcare reform going forward, the experience with MLs suggests that neither an excessively top-down nor bottom-up approach is likely to be desirable. Rather, policymakers will need to consider a combination of national direction with increased local autonomy, to develop mechanisms that would enable policy implementers to evaluate and refine the balance between these two perspectives in a systematic and evidence-based manner over time. For this to work effectively, ongoing cooperation and partnership is required between national and state level stakeholders in the primary healthcare policy space.

In this study MLs had a fairly high level of confidence in their planning function and their ability to explore the needs of their local communities. However, one of the criticisms of the Horvath ${ }^{18}$ review was around duplication of efforts in terms of population health planning and other corporate and engagement activity. We argue that 
this issue is likely to be satisfactorily resolved through the fewer numbers of PHNs that will exist compared to MLs, and that such criticism should not detract from the significant benefits that come from local level needs assessment activities. Lessons from other countries suggest that development of a needs assessment and priority setting can be used effectively by primary healthcare organisations to develop dialogues and communications with key stakeholder groups and also to aid governance and accountability in relation to performance. ${ }^{12}$ Having a robust understanding of the needs of local populations is pivotal to taking an informed approach to organisation and development of services, especially in relation to the achievement of defined equity, access and service outcomes. As the new PHN entities take shape, we would urge them to continue to develop on the work of MLs in fostering needs assessment capability. This should involve the development and use of a strong evidence base to help inform and develop planning, decision-making and stakeholder engagement.

The support and involvement of clinicians is seen as crucial to primary healthcare reform in other jurisdictions (UK, Canada and NZ to name just a few). ${ }^{12} 18$ 24-26 Our survey found a diversity of clinical representation on ML boards and the level of engagement with clinicians varied between MLs. If PHNs are to be successful then engagement and involvement of clinicians in key strategic and decision-making functions is crucial. Respondents in our study were in agreement that stakeholder engagement, and networking in general, are critical to implementation success and a key factor in relation to system integration. However, the difficulty in stakeholder relationships proved to be a significant barrier to reform. Other studies have shown that relationship building can be very difficult for organisations without the right incentives and levers to help shape behaviour. ${ }^{9}{ }^{15}$ MLs, unlike English primary healthcare organisations, had limited access to financial incentives or other levers to drive and influence the commissioning and procurement of services across the system. This point was noted by respondents, who confirmed their lack of power and capacity to incentivise, innovate and reform behaviour. This situation was exacerbated by the current division of roles and responsibilities for healthcare (with States and Territories responsibilities being predominantly hospital based and the Commonwealth government being predominately primary healthcare focused).

This structural aspect further impacted on MLs ability to influence the behaviour of primary healthcare providers and consumers. In building upon the success of the new PHNs, the use of financial incentives should be given close attention, with the understanding that they can be a powerful means of influencing behaviours, provided that they are carefully and compatibly designed. For example, Garber ${ }^{27}$ notes that 'distinct and incompatible incentives among key players can conflict directly with efforts to integrate' ( p.17). An important focus for policymakers engaged in the design and development of meso-level primary healthcare organisations, is to identify the appropriate levers and incentives (financial and other) that can be used to motivate and sustain relationships, collaboration and integration across health services. In this regard, it is noteworthy that a number of countries are looking to joint funding and mixed payment methods as an effective way to incentivise joint working and integration. ${ }^{28} 29$

While structural reform is important, cultures and behaviours will have considerable impact on the way that structural reform is implemented and should not be underestimated. ${ }^{30}{ }^{31} \mathrm{~A}$ recent study by Brown $e t \mathrm{al}^{15}$ identified culture change as a major challenge to MLs objectives around system integration. Culture related to shifting attitudes away from secondary to primary care, and more collaboration rather than silo-based working. ${ }^{15}$

As found in other health systems internationally, implementation of primary care reforms will require effective leadership to navigate the complexity of the health system and the different organisational cultures and current divisions of control and resources. ${ }^{12}$ The PHNs cannot do this alone. They will require the will and support of Commonwealth and State governments and other stakeholder groups including general practitioners.

\section{CONCLUSION}

There is much agreement internationally that a more integrated approach to health services with primary healthcare at the centre is key to the provision of efficient, effective, high quality and sustainable patient centred systems. ${ }^{4} 75$ While this is the aspiration, many difficulties are associated with implementing and realising such integration. This paper has shown that in Australia, MLs have made some positive steps around population-based planning and stakeholder engagement. However, MLs had limited opportunity to establish their role in strengthening primary healthcare and improving integration across the health system. In terms of form and function, our study showed that there was considerable divergence in the way the meso-organisations operated. While this was partly appropriate and enabled the meeting of unique local needs, it was also evident that some of the divergence was unnecessary and detrimental to their establishment. Going forward, we would recommend that there be greater consideration given to national coordination and mechanisms to be used to monitor implementation divergences and facilitate more system-wide harmonisation where appropriate. Ultimately, a balance is required between national policy direction and increased local autonomy.

The importance of understanding the needs of local populations should also not be overlooked or underestimated, a capacity that the MLs had begun to build quite successfully. Our study identified mixed experiences in terms of the relationships forged between MLs and key stakeholders across the Australian healthcare system and that the quality of these relationships was a critical factor 
in the success of primary care reform. This is a very relevant lesson for the new PHNs, who could play a significant role in incentivising and motivating relationships and partnerships between key stakeholders, and thus drive greater integration across the healthcare system overall. However, to achieve this, Commonwealth and State governments have a collaborative role to play in supporting PHNs and ensuring primary care policy objectives are being realised.

Twitter Follow Suzanne Robinson at @Robinsonsuz

Acknowledgements The authors would like to thank those who helped develop and pilot the electronic survey and all those ML employees who took part in the survey. The authors really appreciate the time and effort given over to our research, without such support, studies like this would not be possible.

Contributors SR, VR, DH, PO, and LS designed the study. SR, MD, RV, and LS collected the data. SR, RV, VR, LS analysed the data. SR drafted the manuscript with input from all other authors. All authors approved the final version. SR is guarantor.

Funding The study was funded by Curtin University.

Competing interests None.

Ethics approval Ethical approval for this research was granted from the Curtin University Ethics Committee.

Provenance and peer review Not commissioned; externally peer reviewed.

Data sharing statement No additional data available.

Open Access This is an Open Access article distributed in accordance with the Creative Commons Attribution Non Commercial (CC BY-NC 4.0) license, which permits others to distribute, remix, adapt, build upon this work noncommercially, and license their derivative works on different terms, provided the original work is properly cited and the use is non-commercial. See: http:// creativecommons.org/licenses/by-nc/4.0/

\section{REFERENCES}

1. OECD Health Statistics. How does Australia compare? OECD, 2014.

2. Australian Institute of Health and Welfare. AlHW Cat. No. PHE 6. Health in rural and remote. Canberra, Australia, 1998.

3. National Health and Hospital Reform Commission. A healthier future for all Australians-Final Report. Australian Government, 2009.

4. Ham C, Curry N. Integrated care: what is it? Does it work? What does it mean for the NHS. London: Kings Fund, 2011.

5. WHO. World health report 2008. Primary health care: now more than ever. Geneva: WHO, 2008.

6. Starfield B. Is primary care essential? Lancet 1994;344:1129-33.

7. Suchowersky A, Suchowersky O, Duckett SJ. Can Alberta's primary care networks provide any lessons for Medicare Locals. Med J Aust 2012;196:27-8.

8. Oliver-Baxter J, Bywood P, Brown L. Integrated care: What policies support and influence integration in health care across New Zealand, England, Canada and the United States? PHCRIS Policy Issue Review. Adelaide: Primary Health Care Research \& Information Service, 2013

9. Oliver- Baxter J, Brown L, Bywood P. Integrated care: what strategies and other arrangements support and influence integration at the meso/organisational level? PHCRIS Policy Issue Review.
Adelaide: Primary Health Care Research and Information Service, 2013.

10. Smith J. Curry N, Mays N, et al. Where next for commissioning in the English NHS? London: The Nuffield Trust, 2010.

11. Walshe K, Smith J, eds. Health care management. 2nd edn. Open University Press/McGraw Hill, 2011.

12. Robinson S, Williams I, Freeman $\mathrm{T}$, et al. Priority-setting and rationing in healthcare: evidence from the English experience. Soc Sci Med 2012;75:2386-93.

13. Ham NTC. The quest for integrated health and social care. A case study in Canterbury, New Zealand. London: King's Fund, 2013.

14. McCafferty S, Williams I, Hunter D, et al. Implementing world class commissioning competencies. J Health Serv Res Policy 2012;17 (Suppl 1):40-48I.

15. Brown L, Katterl R, Bywood P, et al. Medicare Locals: a model for primary health care integration? PHCRIS Policy Issue Review. Adelaide: Primary Health Care Research \& Information Service, 2013.

16. Nicholson C, Jackson CL, Marley JE, et al. The Australian experiment: how Primary Health Care Organizations supported the evolution of a primary health care system. J Am Board Fam Med 2012;25:S18-26.

17. Commonwealth of Australia and the States and Territories. The National Health Reform Agreement-National Partnership Agreement on Improving Public Hospital Services. Australian Government, 2011.

18. Horvath J. Review of Medicare Locals. Report to the Minister for Health and Minister for Sport, Australia. Barton: Commonwealth of Australia, 2014.

19. Australian Government Department of Health. Primary Health Networks. http://www.health.gov.au/internet/main/publishing.nsf/ content/primary_health_networks (accessed on 12 Feb 2015).

20. Australian Government Budget 2014-2015. http://www.budget.gov. au/2014-15/content/bp2/html/bp2_expense-14.htm (accessed 12 Feb 2015).

21. Bowling A, Ebrahim S. Handbook of health research methods, investigation measurement and analysis. Washington, DC: National Academies Press, 2005.

22. Australian Institute of Health and Welfare. METReOR Metadata Online Registry Government. http://meteor.aihw.gov.au/content/ index.phtml/itemld/550733 (accessed 12 Feb 2015).

23. Peck E, Dickinson $\mathrm{H}$, Smith J. Transforming or transacting? The role of leaders in organisational transition. Br J Leadership Public Serv 2006;2:4-14.

24. Miller R, Peckham S, Checkland K, et al. Clinical engagement in primary care-led commissioning: a review of the evidence. London: Policy Research Unit in Commissioning and the Healthcare System (PRUComm), 2012

25. Hutchison B, Levesque JF, Strumpf E, et al. Primary health care in Canada: systems in motion. Milbank Q 2011;89:256-88.

26. Thorlby R, Smith J, Barnett $\mathrm{P}$, et al. Learning from New Zealand's independent practitioner associations: primary Care for the $21^{\text {st }}$ century. London: Nuffield Trust, 2012.

27. Garber A. Competition, integration and incentives: the quest for efficiency in the English NHS. London: Nuffield Trust, 2011 issue 2.

28. Rosenthal MB. Beyond pay for performance-emerging models of provider-payment reform. New Eng J Med 2008;359:1197-200.

29. Wranik DW, Durier-Copp M. Physician remuneration methods for family physicians in Canada: expected outcomes and lessons learned. Health Care Anal 2010;18:35-59.

30. Braithwaite J, Westbrook MT, Hindle D. Hospital sector organisational restructuring: evidence of its futility. In: McKee L, Ferlie E, Hyde P, eds. Organizing and Reorganizing: Power and Change in Health Care Organizations. Basingstoke: Palgrave Macmillan, 2008:33-45.

31. Hunter D. Change of government: one more big band health care reform in England's National Health Service. Int J Health Serv 2011;41:159-74. 